\title{
PIV measurement of tube-side in a shell and tube heat exchanger
}

\author{
Kai Wang ${ }^{1 *}$ Zixu Zhang ${ }^{1}$, Qiong $\mathrm{Liu}^{2}$, Xincheng $\mathrm{Tu}^{3}$, Hyoung-Bum Kim ${ }^{4,}$ 5* $^{*}$ \\ ${ }^{1}$ Jiangsu University, Research Center of Fluid Machinery Engineering and Technology, Zhenjiang 202013, China \\ ${ }^{2}$ Jiangsu University, Library, Zhenjiang 202013, China \\ ${ }^{3}$ Midea Kitchen Appliances Division, Foshan 528311, China \\ ${ }^{4}$ Gyeonsang National University, Research Center for Aircraft Parts Technology, Jinju 660701, S. Korea \\ ${ }^{5}$ Gyeonsang National University, School of Mechanical and Aerospace Engineeeing, Jinju 660701, S. Korea \\ "Corresponding authors: e-mail: wangkai@ujs.edu.cn; kimhb@gnu.ac.kr
}

\begin{abstract}
In order to improve the performance of the shell and tube heat exchanger, a porous baffle and a splitter bar are employed in this research. Through the arrangement of the porous baffle in the tube-side inlet and the splitter bar in the tube, the flow distribution of liquid in the heat exchanger is improved. PIV technology is used to investigate the unsteady flow in the tube-side inlet and the outlet of different models. The porous baffle significantly improves the flow of fluid in the shell and tube heat exchanger, especially by eliminating/minimizing the maldistribution of fluid flow in the tube-side inlet. The performance of the arc baffle is better than that of the straight baffle. The splitter bar has a minimal effect on the flow field of the tube-side inlet, but it effectively improves the flow in the tube bundle and restrains the vortex generation in the tube-side outlet.
\end{abstract}

Keywords: shell and tube heat exchanger, tube-side, PIV, unsteady flow.

\section{INTRODUCTION}

The heat exchanger is a device that transfers part of the heat of the hot fluid to the cold fluid. Especially in the chemical industry, the heat exchanger can be used as a heater, cooler, condenser, evaporator, reboiler, and so on. In the modern chemical industry, the investment in heat exchangers accounts for about $30 \%$ of the total equipment investment and about $40 \%$ of the total processing equipment investment of refineries. Furthermore, the sea water desalination device is almost entirely composed of heat exchangers. As the demand for heat exchangers in several large industries such as the chemical, shipbuilding, food, and pharmaceutical industry continuously grows, the heat exchanger industry will maintain steady development in the future. The shell and tube heat exchanger still occupies a major market share because of its robustness and economical price.

In order to improve the thermal performance, many scholars have studied the design of shell and tube heat exchangers. Pan et al. ${ }^{1}$ focused on the progress of the traditional heat intensification technology, including tube-side enhancements and shell-side enhancements, and compared the influence of these technologies on the heat transfer performance and available substantial economic benefits. Research results show that the high efficiency heat exchangers with combining intensification techniques are able to achieve significant economic benefit of exchanger design. Sanaye et al. ${ }^{2}$ chose the maximum effectiveness and the minimum cost of heat transfer as objective functions of a genetic algorithm to optimize the heat exchanger and obtained the Pareto optimal solution of the tube arrangement, tube diameter, tube pitch ratio, tube length, tube number, baffle spacing ratio, and baffle cut ratio. The results revealed the level of conflict between two objective functions. Tube pitch ratio, tube length, and tube number as well as baffle spacing ratio are important design parameters that caused a conflict between the effectiveness and total cost. Ozden et al. ${ }^{3}$ numerically studied the influence of baffle spacing, baffle cut, and shell diameter on the heat transfer coefficient and the pressure drop with numerical simulations. They observed that the simulation results are in accordance with the Bell-Delaware results. Hosseini et al. ${ }^{4}$ compared the performance of three tube bundles with different inner surface (smooth, corrugated, and with micro-fins). They found that corrugated and micro-fin tubes showed degraded performance at a Reynolds number below a certain value $\left(R_{\mathrm{e}}<400\right)$ and the performance of the heat exchanger greatly improved for micro-finned tubes at a higher Reynolds number. Pacio et $a .^{5}$ found that the parallel tube bundle flow nonuniformity will seriously affect the performance of the heat exchanger, especially the two-phase flow, such as the flow in the evaporator. Mohr et al. ${ }^{6}$ presented the design equations for the inlet section of heat exchangers with variations of a broad range of geometrical parameters, e.g., tube pitch, shell diameter, nozzle diameter, span width, distance between nozzle exit, and tube bundle. Kim et al. ${ }^{7}$ studied the uniform distribution of gas phase flow in a shell and tube heat exchanger by numerical simulation and found that uniformity of the flow distribution would increase with the header length, whereas it decreases with the gas flow rate. Wang et al. ${ }^{8-9}$ used a numerical optimization method to find the optimized porous baffle in the tube side inlet of a shell and tube heat exchanger with a minimum average absolute error of the flow rate and carried out an experimental validation study of the optimized baffle. The results showed that the flow uniformity was improved using the optimized model.

Research on the shell and tube heat exchanger is now being concentrated on the shell-side design. Although a numerical simulation can predict the performance and pressure drop of the heat transfer, experimental measurement is very important ${ }^{10-12}$. Due to the complex flow path in the tube side, it will cause many kinds of fluid vortex, vibration, and acoustic resonance. Therefore, the purpose of this study is to explore the flow distribution of the inlet and outlet of the tube side. 


\section{EXPERIMENT PLATFORM AND MEASUREMENT METHODS}

\section{Test bench}

Figure 1 shows the test bench of the shell and tube heat exchanger. It mainly includes the shell and tube heat exchanger, flow meter, pump, and water tank. The shell and tube heat exchanger in this experiment is a $1 / 3$ scaled-down model of the LNG evaporator. The inlet diameter and outlet diameter of the shell and tube heat exchanger are $80 \mathrm{~mm}$. The flow rate in the experiment is 100 litres per $\min (\mathrm{lpm})$.

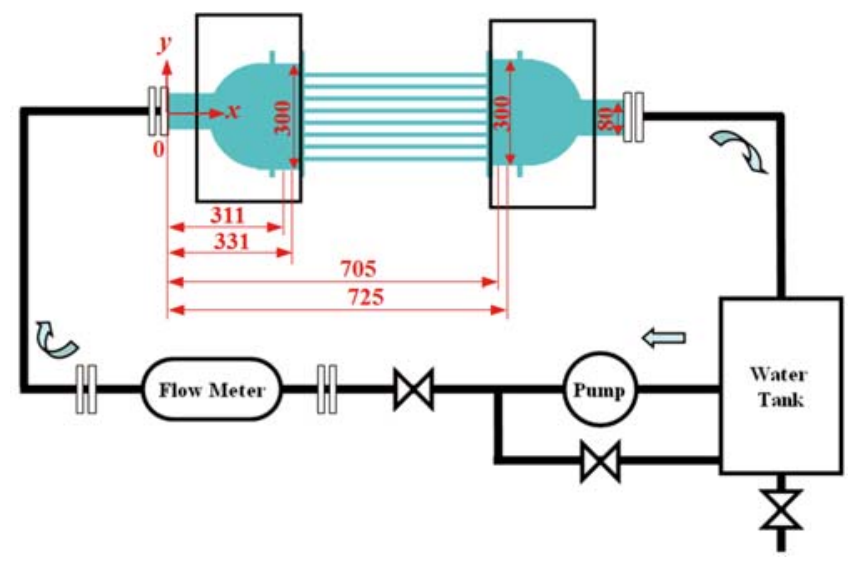

Figure 1. Test bench

\section{Research model}

Figure 2 shows three-dimensional diagrams of all the models, including three sets of the tube-side inlet and two sets of the tube bundle and the tube-side outlet. Figure 3(a) shows the diameters and the positions of the holes on the straight baffle. The straight baffle was designed based on plain design knowledge. The porosity of the baffle was chosen as $30 \%$ to avoid the separation bubble behind the baffle location. Diameters of circular holes in the straight baffle are $5 \mathrm{~mm}, 8 \mathrm{~mm}$ and 10 $\mathrm{mm}$ along the radial direction. The numbers of these three circular holes are 52, 108, and 156, respectively. The parameters of holes in the arc baffle are shown in Figure 3(b). The arc baffle was designed based on the CFD optimization method ${ }^{8}$. The porosity of the baffle was chosen to improve the flow distribution. Diameters
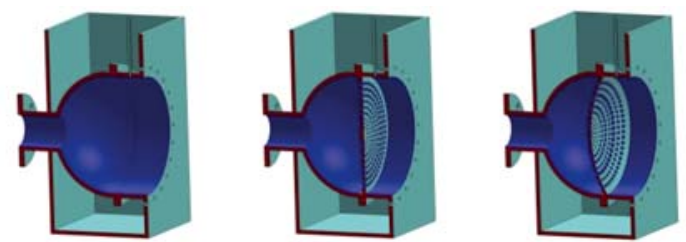

(a) Inlet without baffle (b)Inlet with straight baffle (c) Inlet with arc baffle
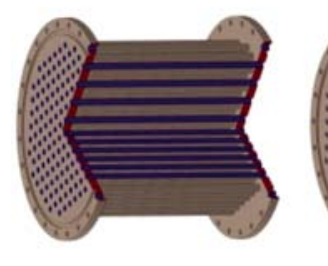

(d) Tube without bar

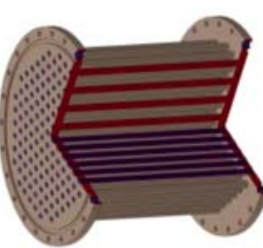

(e) Tube with bar

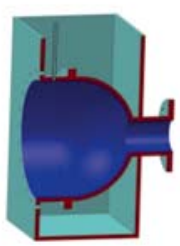

(f) Outlet of circular holes in the arc baffle are $3.7 \mathrm{~mm}, 4.9 \mathrm{~mm}$, $4.2 \mathrm{~mm}, 4.9 \mathrm{~mm}, 4.3 \mathrm{~mm}, 8 \mathrm{~mm}, 9.1 \mathrm{~mm}, 7.6 \mathrm{~mm}$, $11.3 \mathrm{~mm}, 8.2 \mathrm{~mm}$, and $9.1 \mathrm{~mm}$ along the radial direction. Numbers of the circular holes are 1, 8, 12, 16, 24, 28, $36,44,44,52$, and 60 , respectively.

The diameter of 148 tubes is $10 \mathrm{~mm}$ and the distance between two adjacent tubes is $22 \mathrm{~mm}$, as shown in Figure 3(c). In Figure 3(d), the width of the splitter bar is $3 \mathrm{~mm}$ and the bar is located in the middle position of the tubes. This model has a reduced number of tube bundles compared with the proto-type shell and tube heat exchanger, while the width of the splitter bar is $1 / 3$ scaled-down compared to the proto-type shell and tube heat exchanger.

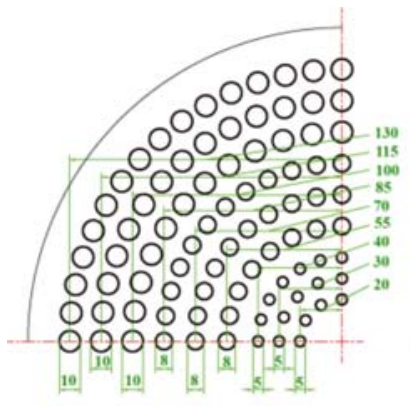

(a) Straight baffle cut

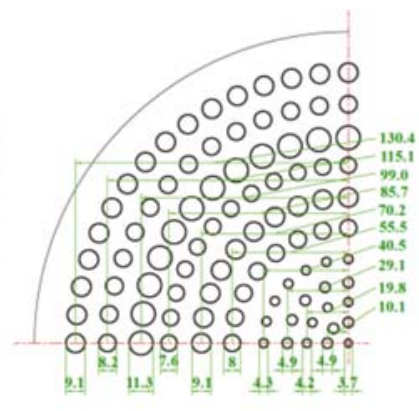

(b) Arc baffle cut

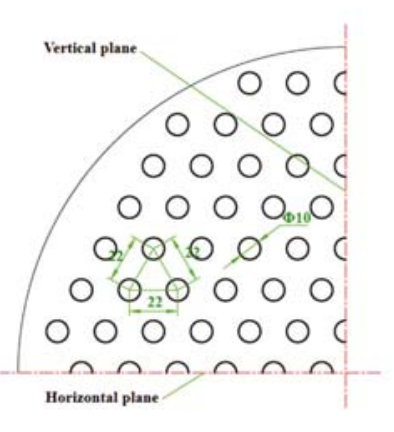

(c) Tube cut without bar

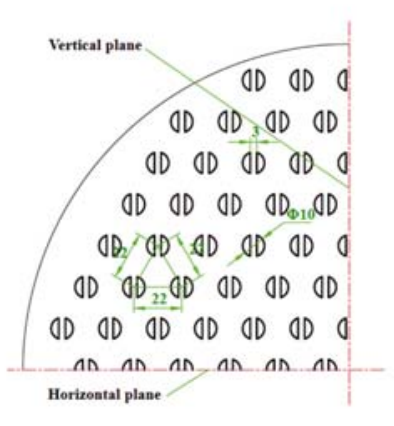

(d) Tube cut with bar
Figure 3. Sketch of baffle cuts and tube cuts

\section{PIV processing method}

A CCD camera with $1.6 \mathrm{k} \times 1.2 \mathrm{k}$ pixel resolution was used to measure the instantaneous velocity fields around the tube-side of the shell and tube heat exchanger. In total, 400 instantaneous velocity vector fields were adopted to obtain their mean properties with the ensemble averaging method. The cross-correlation based PIV method with window offsetting was used to increase the spatial resolution of the instantaneous velocity vectors. The image processing and PIV calculation were conducted by using Insight $3 \mathrm{G}$ software. The cross-correlation method was applied and the interrogation window size was $16 \times 16$ pixels with $50 \%$ overlap. In this experiment, we applied silver coated hollow glass spheres as seeding particles. Their density is $1.65 \mathrm{~g} / \mathrm{cm}^{3}$. A Nd:YAG laser sheet with a wavelength of $532 \mathrm{~nm}$ illuminated the flow field at a specific cross section. The sampling frequency of the PIV system is $15 \mathrm{~Hz}$.

In this PIV experiment, we measured the inlet velocity of the tube-side in the shell and tube heat exchanger and

Figure 2. Research models 
calculated the flow rate of the inlet, which has a value of $101.26 \mathrm{lpm}$. However, the average flow rate in the flow meter is $100.11 \mathrm{lpm}$. The relative difference between the flow rate from the PIV and the flow meter is $1.15 \%$ and thus the uncertainty of mean velocity data is $1.15 \%$.

\section{MEASUREMENT RESULTS AND ANALYSIS}

\section{Mean velocity vector field}

Figure 4 is the mean velocity vector field of the tube-side inlet of the shell and tube heat exchanger without a porous baffle. Because laser light illumination is blocked, information for this blocked region was not obtained. Figures 4(a) and 4(b) demonstrate the flow pattern of the vertical and horizontal planes of the model without a splitter bar. It can clearly be seen that the flow streamlines changed after impacting the tube sheet. The vector direction of velocity deviates from the center line, and is shifted to the outside of the radial direction. The inertial force of the fluid is greater than the sticking force, resulting in a relatively large reverse vortex. Since the distribution of the tube bundle is not completely symmetrical, the shape and the position of the vortex on the vertical and horizontal planes are not the same. Comparison of their location shows that the distances between the vortex cores and central axis are roughly the same. However, in the axial position, the vortex core in the vertical plane is closer to the tube sheet than in the horizontal plane. Due to the mutual impact of the fluid and the vortex, the reverse flow and the stationary flow can be easily found near the wall. The non-uniform flow will affect the flow rate and velocity of the fluid through the tube bundle, which will lead to a change of the heat transfer efficiency of the heat exchanger.
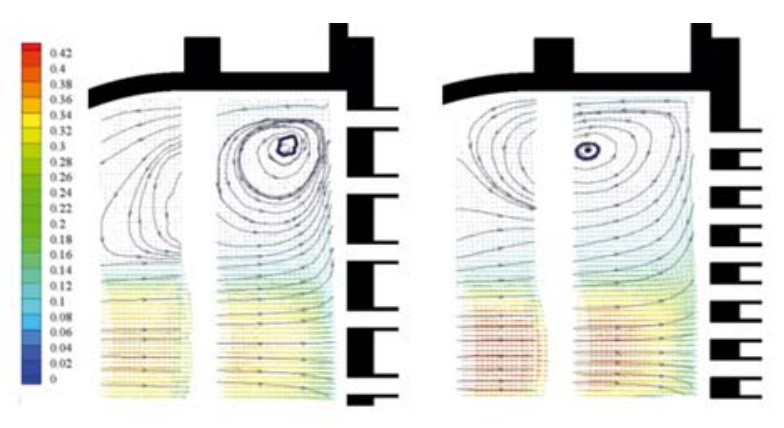

(a) Vertical plane without bar

(b) Horizontal plane without bar

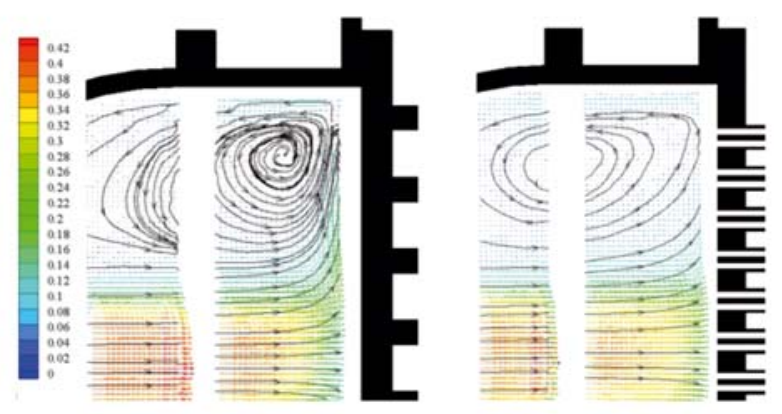

(c) Vertical plane with bar

(d) Horizontal plane with bar

Figure 4. Velocity flow fields of tube-side inlet without baffle (Unit: $\mathrm{m} / \mathrm{s}$ )
Figures 4(c) and 4(d) present the mean velocity field of the vertical and horizontal planes of the model with a splitter bar. It can be seen that the addition of the splitter bar has no obvious influence on the shape and size of the vortex. However, compared with Figures 4(a) and 4(b), some changes have taken place in the position of the vortex. It is obvious that the center of the vortex is moving away from the tube sheet. The center of the vortex is blocked in the horizontal plane. It can be inferred that the radial position of the center is the same as that of the vertical plane, which is also the same as the model without a splitter bar.

Figure 5 gives the mean velocity vector field of the tube-side inlet with a straight baffle. The relatively large reverse vortex in Figure 4 is divided into some small vortices due to the addition of the straight baffle. The vortex on the left side of the baffle is larger and the flow distribution is uneven. After flowing through the baffle, the velocity of fluid decreases, and the streamline on the right side of the straight baffle tends to be axial plane. The fluid passes through the baffle hole, resulting in the formation of small vortices, which have very little effect on the velocity direction. In addition, in the corner between the tube sheet and the wall, a large vortex is formed due to the impact of the fluid on the tube sheet, which affects the flow pattern around the vortex. However, compared with the vortex formed in the model without the baffle, it becomes smaller. Therefore, we found that the porous baffle can effectively suppress the vortex and reduce the resulting head loss. In addition, it can be found from Figure 5 that at the corner of the vertical and horizontal planes, the positions of these vortices are basically the same.

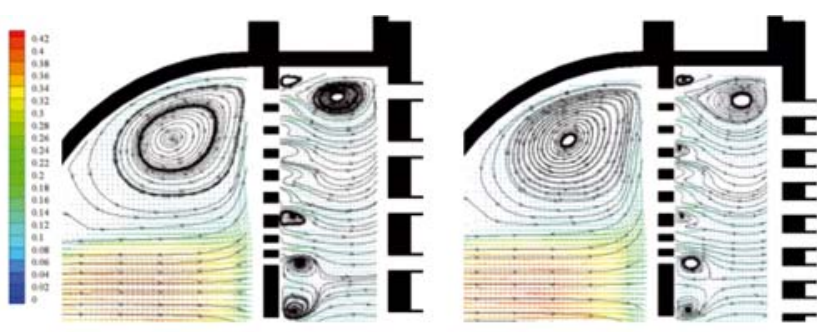

(a) Vertical plane without bar

(b) Horizontal plane without bar
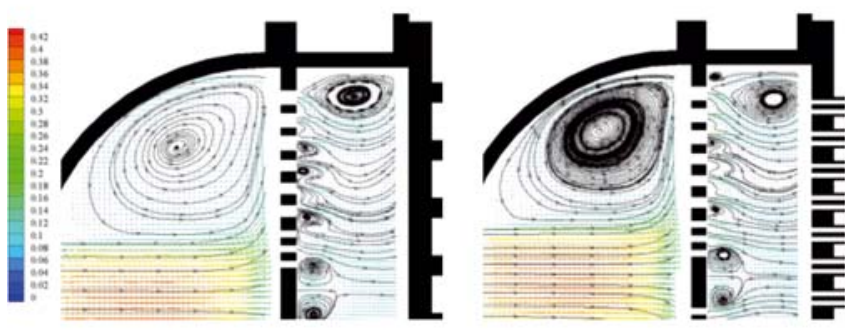

(c) Vertical plane with bar

(d) Horizontal plane with bar

Figure 5. Velocity flow fields of tube-side inlet with straight baffle (Unit: $\mathrm{m} / \mathrm{s}$ )

The mean velocity fields of the model with the arc baffle are shown in Figure 6. It can be found that the vortex in the corner between the tube sheet and the wall becomes smaller, and the core of the vortex deviates from the side of the tube sheet. The flow on the right side of the vortex tends to be uniform and the overall streamlines are parallel to the central axis. Because of the blocking of laser light illumination, some informa- 


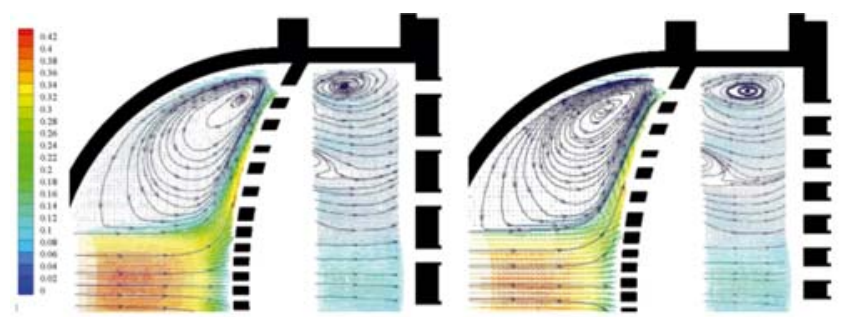

(a) Vertical plane without bar

(b) Horizontal plane without bar
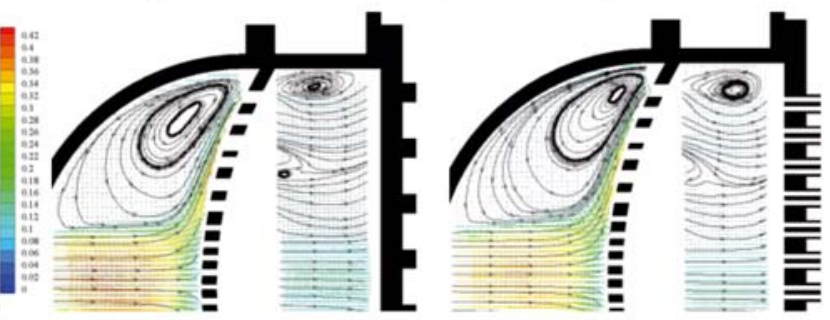

(c) Vertical plane with bar

(d) Horizontal plane with bar

Figure 6. Velocity flow fields of tube-side inlet with arc baffle (Unit: $\mathrm{m} / \mathrm{s}$ )

tion behind the arc baffle did not appear. From the part that can be observed, the arc baffle has the best inhibition effect on the vortex and can make the fluid enter the tube bundle uniformly, and thus effectively enhances the even flow distribution and increases the heat transfer efficiency.

In order to further verify and analyze the influence of the arc baffle, straight baffle, and bar on the flow distribution in the tube bundle, we obtained the mean velocity vector field of the tube-side outlet with PIV. Figure 7 shows the flow pattern at the tube-side outlet without a porous baffle. The number of tubes on the horizontal plane is more than that on the vertical plane, and the difference in the velocity distribution on the two planes is hence also very large. The vortices on the two
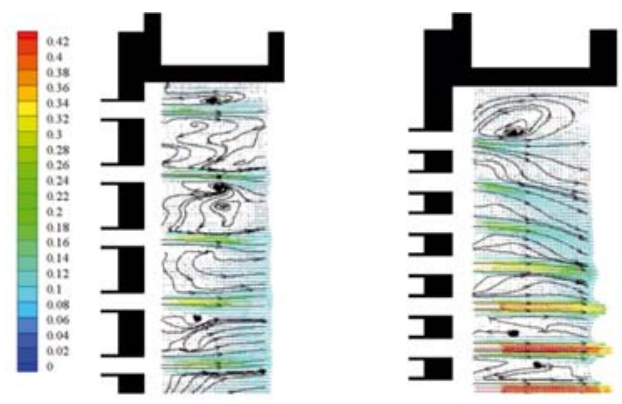

(a) Vertical plane without bar

(b) Horizontal plane without bar
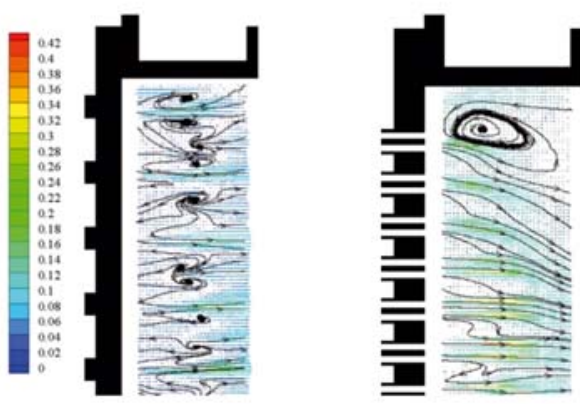

(c) Vertical plane with bar

(d) Horizontal plane with bar

Figure 7. Velocity flow fields of tube-side outlet without baffle (Unit: $\mathrm{m} / \mathrm{s}$ ) planes basically occur in the corner between the tube sheet and the wall.

The flow behind each pipe is very uniform. Due to the distribution of the tube bundle, the vortex on the horizontal plane is also relatively much larger as well. From the vertical plane, in addition to the region near the tube sheet, the other flow field is very messy and there are many smaller vortices and a large amount of reverse flow. It can also be found that the fluid velocity of the horizontal plane is much larger than that of the vertical plane and the difference between the central velocities is more obvious. The impingement region of the vertical plane at the tube-side inlet is larger than that of the horizontal plane. Therefore, the influence of the vortex on the flow velocity is larger. The flow near the axis of the vertical plane with higher velocity will lead to lower pressure. This phenomenon is more obvious after adding a splitter bar, which is shown in Figures 7(c) and 7(d). It can be seen that the velocity of the fluid is reduced and the inertial force of the fluid decreases when the bars are added. The flow pattern of the vertical plane is further disordered and some small vortices are formed. The phenomenon that fluid on the horizontal plane gathers toward the center is more obvious. As observed from this phenomenon, before adding the splitter bar, the distribution of velocity in the tube bundle is uneven. The flow near center is very fast, which will affect the overall heat transfer efficiency.

Figure 8 is the mean velocity vector field of the tube-side outlet with a straight baffle. Compared with Figure 7, an obvious change is found that the overall velocity of the fluid decreases. The uneven distribution of velocity has been improved, and the velocity difference with different radiuses is reduced. When the fluid flows out of the heat exchanger through the tube bundle, the flow streamlines are basically parallel to each other. Nonetheless, the large decrease of flow velocity will affect the heat transfer efficiency of the heat exchanger.
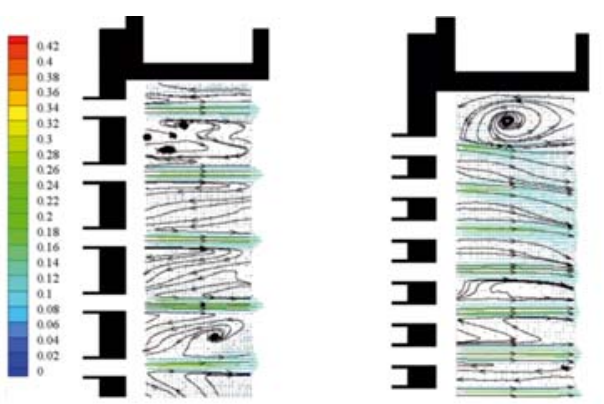

(a) Vertical plane without bar

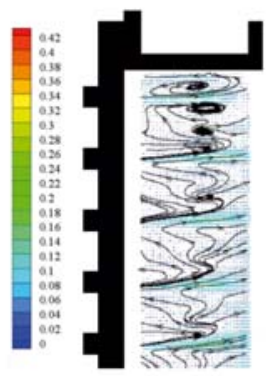

(b) Horizontal plane without bar

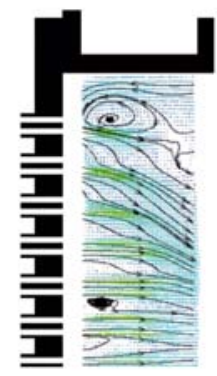

(c) Vertical plane with bar

(d) Horizontal plane with bar

Figure 8. Velocity flow fields of tube-side outlet with straight baffle (Unit: $\mathrm{m} / \mathrm{s}$ ) 
Figure 9 is the mean velocity vector field of the tube-side outlet with an arc baffle. Compared with Figure 7, the flow distribution on the vertical plane is improved, and the fluid velocity near the center is consistent with other positions. Compared with Figure 8, after adding the splitter bar, the vortex in the corner between the tube sheet and the wall is reduced and the flow velocity near the tube sheet is larger. It can be inferred that the flow velocity in the tube bundle of the model with the arc baffle is larger than that with the straight baffle.
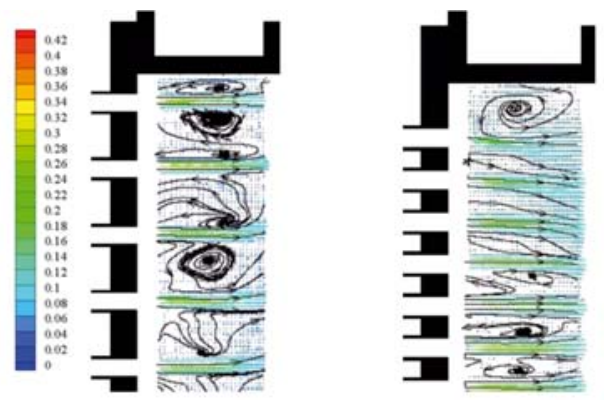

(a) Vertical plane without bar

(b) Horizontal plane without bar
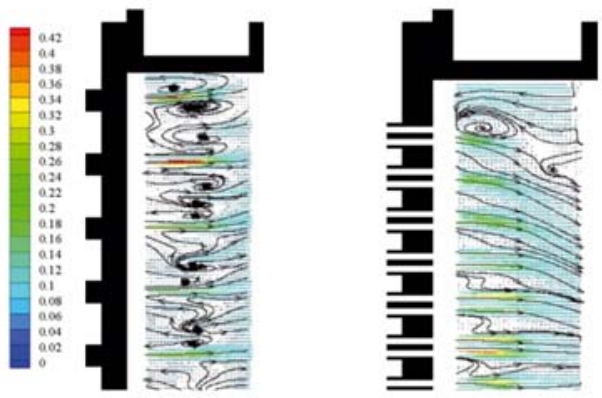

(c) Vertical plane with bar

(d) Horizontal plane with bar

Figure 9. Velocity flow fields of tube-side outlet with arc baffle (Unit: $\mathrm{m} / \mathrm{s}$ )

\section{Velocity curves and cumulative flow rate curves}

In order to further analyze the influence of porous baffle and splitter bar on the flow distribution, we extracted the data at locations $311 \mathrm{~mm}, 331 \mathrm{~mm}, 705 \mathrm{~mm}$, and $725 \mathrm{~mm}$ away from the left boundary of the tube-side inlet (shown in Figure 1). The curves show the flow distribution along the radius of vertical and horizontal planes. Figure 10 shows the axial velocity and flow rate of fluid in the tube-side inlet. The purple solid lines represent the axial velocity of the ideal uniform flow and the theoretical cumulative flow rate. Figures 10(a) and 10 (b) are the axial velocity and cumulative flow rate of the section at $311 \mathrm{~mm}$. The profiles of the three models with/without a bar on the vertical and horizontal planes is similar, while the effect of the porous baffle on the flow is very obvious. The straight baffle and arc baffle are helpful in reducing the velocity difference and improving the flow field of the tube-side inlet.

The velocity difference between flow in the axis and the near wall is very large and the velocity in the axis is $0.35 \mathrm{~m} / \mathrm{s}$, which is much larger than the theoretical velocity. The flow velocity near the wall $(r>0.1 \mathrm{~m})$ is not only smaller than the other two models, there are some differences with the theoretical velocity. This leads to a great decrease of the heat transfer performance of the tubes near the wall. After adding the porous baffle, the

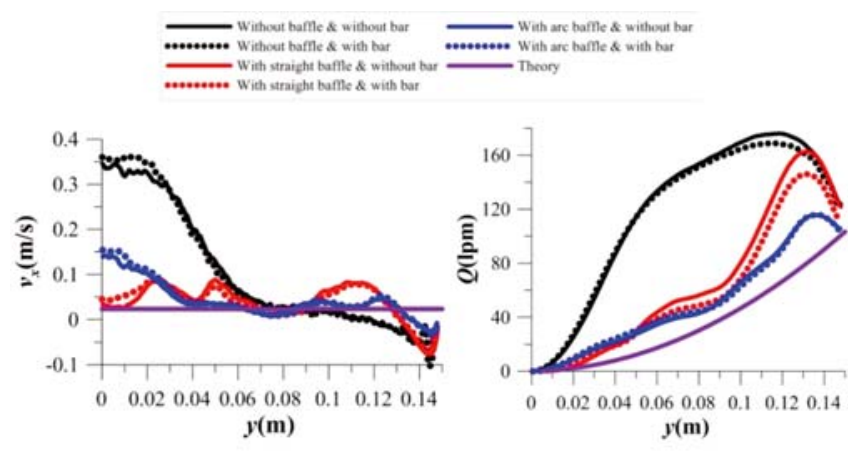

(a) Vertical plane at the distance of $311 \mathrm{~mm}$
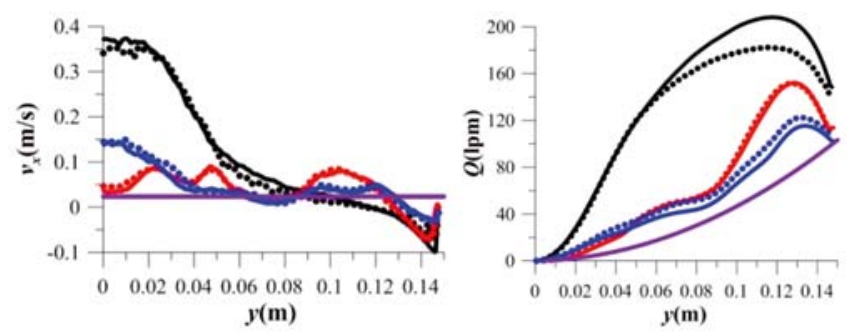

(b) Horizontal plane at the distance of $311 \mathrm{~mm}$
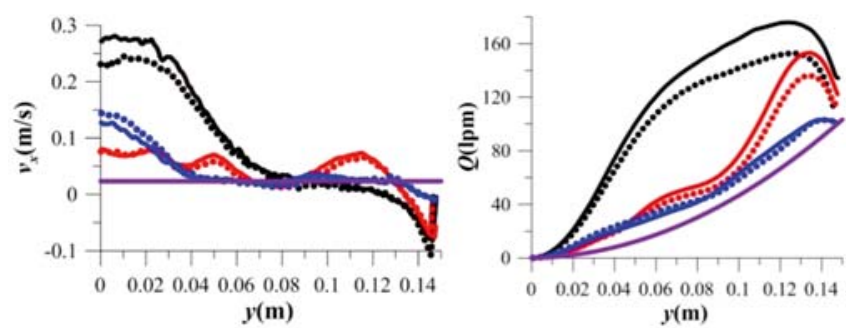

(c) Vertical plane at the distance of $331 \mathrm{~mm}$
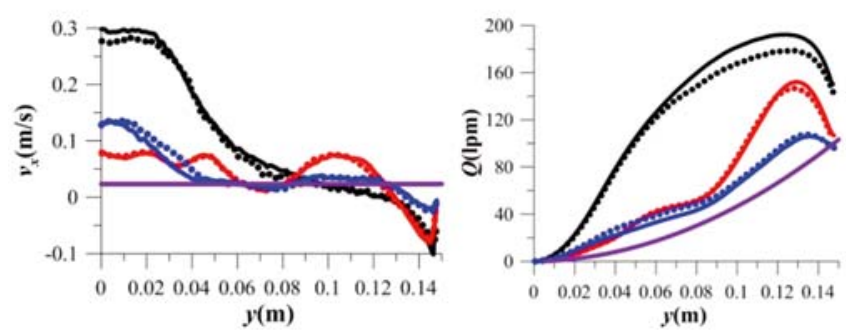

(d) Horizontal plane at the distance of $331 \mathrm{~mm}$

Figure 10. Velocity curves and flow rate curves of tube-side inlet

fluctuation of the axial velocity curve decreases obviously. The performance of the arc baffle is better and the flow velocity $(r>0.04 \mathrm{~m})$ is closer to the theoretical curve. The velocity of the model with the straight baffle is less than that with the arc baffle in the central area. The main reason is that there is no hole in the area $(\mathrm{r}<0.02 \mathrm{~m})$ in the straight baffle, while the speed is obviously small. In the comparison of negative area on the left of Figure $10(\mathrm{a})$, the absolute value of negative velocity of the arc baffle is the smallest. It can be seen that the flow rate curve with the arc baffle is closest to the theoretical curve. Furthermore, there is a considerable degree of wave crest, and the maximum value of the wave is 1.6-2 times larger than the theoretical flow rate. Comparing Figure 10, it can be found that the influence of distance away from the baffle and bar in the tube bundle on the axial velocity distribution of the tube-side inlet is small in both cases. 
Vertical plane without bar - Horizontal plane without har

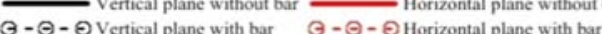
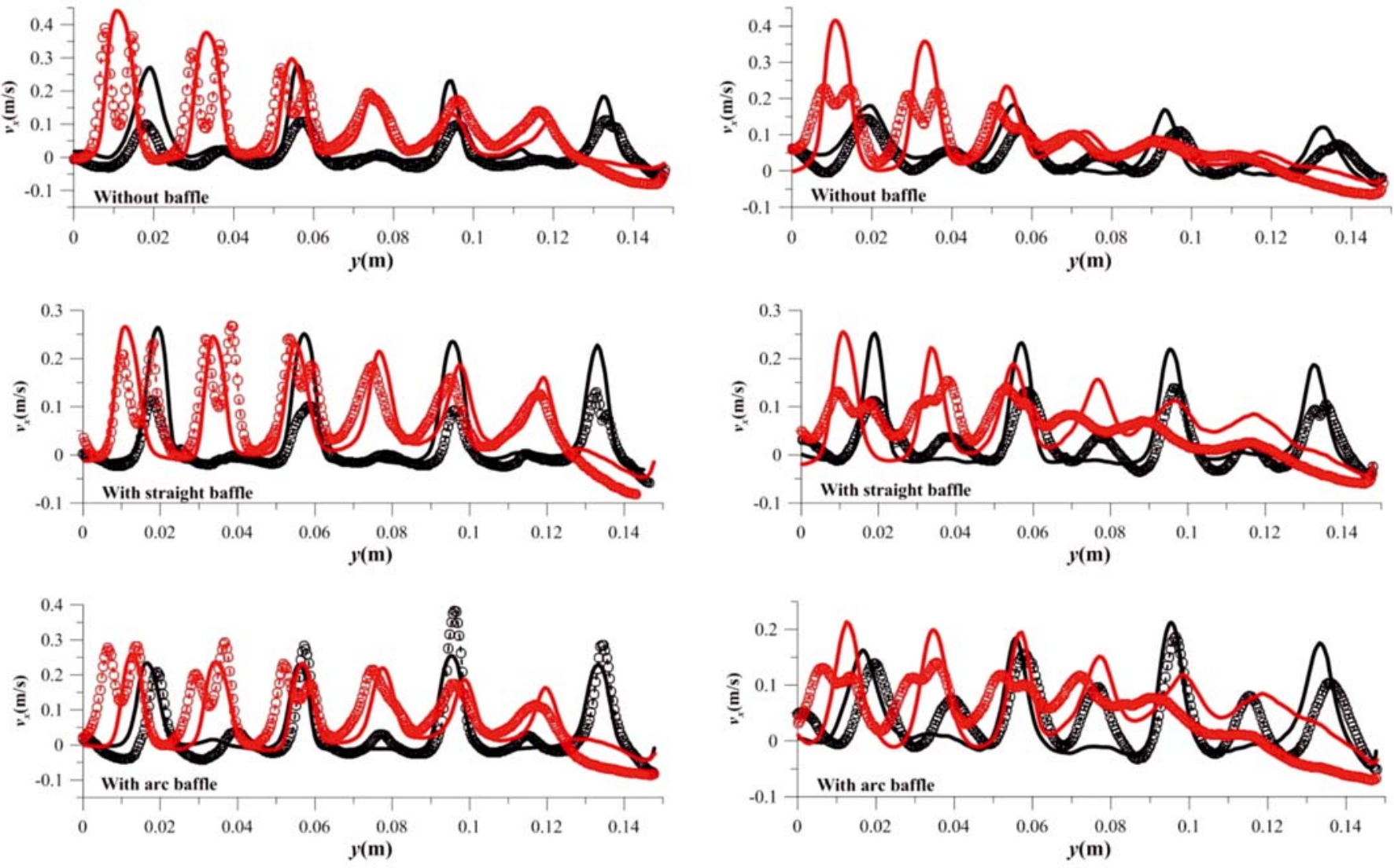

(a) The distance of $705 \mathrm{~mm}$

(b) The distance of $725 \mathrm{~mm}$

Figure 11. Velocity curves of tube-side outlet

Figure 11 shows the axial velocity of fluid in the tube-side outlet. Two sections at $705 \mathrm{~mm}$ and $725 \mathrm{~mm}$ were selected. Comparing the three models in Figure 11(a) reveals that the fluctuation of the axial velocity curve of the model with the porous baffle is much less than that without the baffle. Due to the location of $705 \mathrm{~mm}$ being very near to the outlet of the tube, many vortices are generated and the flow is very complex, and therefore the effect of the different baffles, straight and arc, is not apparent. The influence of the porous baffle and the bar can clearly be seen from Figure 11(b). Comparing the horizontal plane, the maximum velocity value of the model without the porous baffle is $0.4 \mathrm{~m} / \mathrm{s}$. After adding the straight baffle, this value dropped to $0.25 \mathrm{~m} / \mathrm{s}$. The value of the model with the arc baffle is $0.21 \mathrm{~m} / \mathrm{s}$. After adding the bar, this value is further reduced to $0.15 \mathrm{~m} / \mathrm{s}$. It can be found that the effect of the porous baffle and the splitter bar on improving the flow distribution is obvious.

\section{CONCLUSIONS}

In this paper, the velocity fields of the tube-side inlet and outlet with three models in the shell and tube heat exchanger were measured with PIV technology.

Porous baffles can significantly improve the flow distribution, especially the maldistribution of fluid flow in the tube-side inlet. The baffles cause the liquid to flow smoothly into the tube bundle and reduce the impact loss at the tube sheet.
The velocity fluctuation of the model with an arc baffle is less than that with a straight baffle and the velocity curve of the model with the arc baffle is closer to the theoretical velocity curve. The arc baffle thus provides the best rectifying performance.

The splitter bar effectively improves the flow distribution in the tube bundle, and restrains the vortex generation on the tube-side outlet. By reducing the area of the flow dead zone of the outlet, the thermal efficiency of the shell and tube heat exchanger will be improved.

\section{ACKNOWLEDGEMENT}

The work was supported by the National Key Research and Development Program of China (Grant No. 2016YFB0200901), the Basic Science Research Program through the National Foundation of Korea (NRF) funded by the Ministry of Education (Grant No. 2015R1D1A1A01059272), and Priority Academic Program Development of Jiangsu Higher Education Institutions of China (PAPD).

\section{LITERATURE CITED}

1. Pan M., Jamaliniya S., Smith R., Bulatov I. \& Goughb M., Higleyb T. \& Droegemuellerb P. (2013). New insights to implement heat transfer intensification for shell and tube heat exchangers. Energy 57(8), 208-221. DOI: 10.1016/j.energy.2013.01.017.

2. Sanaye, S. \& Hajabdollahi, H. (2010). Multi-objective optimization of shell and tube heat exchangers. Appl. Therm. 
Enginee. 30(14), 1937-1945. DOI: 10.1016/j.applthermaleng.2010.04.018.

3. Ozden, E. \& Tari, I. (2010). Shell side CFD analysis of a small shell-and-tube heat exchanger. Energy Conver. Managem. 51(5), 1004-1014. DOI: 10.1016/j.enconman.2009.12.003.

4. Hosseini, R., Hosseini-Ghaffar, A. \& Soltani M. (2007). Experimental determination of shell side heat transfer coefficient and pressure drop for an oil cooler shell-and-tube heat exchanger with three different tube bundles. Appl. Therm. Enginee. 27(5-6), 1001-1008. DOI: 10.1016/j.applthermaleng.2006.07.023.

5. Pacio, J.C. \& Dorao, C.A. (2010). A study of the effect of flow maldistribution on heat transfer performance in evaporators. Nuc. Enginee. Design 240(11), 3868-3877. DOI: 10.1016/j.nucengdes.2010.09.004.

6. Mohr, U. \& Gelbe, H. (2000). Velocity distribution and vibration excitation in tube bundle heat exchangers. Int. J. Therm. Sci. 39(3), 414-421. DOI: 10.1016/S1290-0729(00)00214-3.

7. Kim, M.I., Lee, Y., Kim B.W., Lee, D.H. \& Song W.S. (2009). CFD modeling of shell-and-tube heat exchanger header for uniform distribution among tubes. Korean J. Chem. Enginee. 26(2), 359-363. DOI: 10.1007/s11814-009-0060-7.

8. Wang, K., Tu, X.C., Bae, C.H. \& Kim, H.B. (2015). Optimal design of porous baffle to improve the flow distribution in the tube-side inlet of a shell and tube heat exchanger. Int. J. Heat Mass Trans. 80, 865-872. DOI: 10.1016/j.ijheatmasstransfer.2014.09.076.

9. Wang, K., Tu, X.C. \& Kim, H.B. CFD simulation and PIV measurement of a shell and tube heat exchanger. The 9th Pacific Symposium on Flow Visualization and Image Processing, 25-28 August 2013. Busan, Korea.

10. Wang S., Wen, J. \& Li, Y. (2009). An experimental investigation of heat transfer enhancement for a shell-and-tube heat exchanger. App. Therm. Enginee. 29(11-12), 2433-2438. DOI: 10.1016/j.applthermaleng.2008.12.008.

11. Lalot, S., Florent, P., Lang, S.K. \& Bergles, A.E. (1999). Flow maldistribution in heat exchangers. Appl. Therm. Enginee. 19(8), 847-863. DOI: 10.1016/S1359-4311(98)00090-8.

12. Jiao, A., Zhang, R. \& Jeong, S. (2003). Experimental investigation of header configuration on flow maldistribution in plate-fin heat exchanger. Appl. Therm. Enginee. 23(10), 1235-1246. DOI: 10.1016/S1359-4311(03)00057-7. 